This Technical Report is also published as Chapter 43 IN

M-Health: Emerging Mobile Health Systems, Robert H. Istepanian, Swamy Laxminarayan, Constantinos S. Pattichis, Editors, 2006.

Springer. $624 \mathrm{p}$.

ISBN: 0-387-26558-9.

\begin{abstract}
AS
REMOTE MONITORING FOR HEALTHCARE AND FOR SAFETY IN EXTREME ENVIRONMENTS

Val Jones*, Nadav Shashar, Oded Ben Shaphrut, Kevin Lavigne, Rienk Rienks, Richard Bults, Dimitri Konstantas, Pieter Vierhout, Jan Peuscher, Aart van Halteren, Rainer Herzog and Ing Widya
\end{abstract}

* Val Jones, Centre for Telematics and Information Technology/Faculty of Electrical Engineering, Mathematics and Computer Science, University of Twente, PO Box 217, 7500 AE, Enschede, The Netherlands, v.m.jones@ewi.utwente.nl. 


\title{
REMOTE MONITORING FOR HEALTHCARE AND FOR SAFETY IN EXTREME ENVIRONMENTS
}

\author{
Val Jones*, Nadav Shashar, Oded Ben Shaphrut, Kevin Lavigne, Rienk \\ Rienks, Richard Bults, Dimitri Konstantas, Pieter Vierhout, Jan \\ Peuscher, Aart van Halteren, Rainer Herzog and Ing Widya.
}

\section{INTRODUCTION}

In this chapter we examine the potential use of remote health monitoring using Body Area Networks (BANs) to support individuals who are working or pursuing recreational activities in extreme environments.

Monitoring can be defined as (periodic or continuous) measurement and observation of dynamically changing physical attributes of a system. The observing agent may be a human or may be another system. A monitoring system may be coupled with a control system, where output of sensors is used to effect changes to the system via a feedback control loop. To give a simple example, a central heating control system incorporates monitors (temperature sensors) whose readings are used as feedback to control the operation of the thermostat, which effects changes in the process such as switching the pump and/or heating element off and on. Sensors simply 'listen', whilst actuators cause some physical effect such as activating a pump.

In health monitoring the monitored systems are human physiological systems and the attributes measured are variable parameters of those systems. An example would

* Val Jones, Centre for Telematics and Information Technology/Faculty of Electrical Engineering, Mathematics and Computer Science, University of Twente, PO Box 217, 7500 AE, Enschede, The Netherlands, v.m.jones@ewi.utwente.nl. 
be monitoring of the functioning of the cardiovascular system by measuring ECG, oxygen saturation and blood pressure. In remote monitoring the sensor data is transmitted to a distant location for some kind of processing.

A combination of technological developments have made remote monitoring of physiological signals a feasible option in healthcare. These advances include: development of low-cost lightweight miniature biosensors; new non-invasive and minimally invasive measurement techniques; small but powerful mobile devices incorporating both computation and communication functionality (e.g. programmable mobile phones, PDAs with telecommunications extensions, computercell phone hybrids); and advances in wireless communication technologies (both short and long range).

Benefits associated with remote patient monitoring include enhanced freedom for the patient, reduced demand for hospital beds and the ability to control situations remotely location in order to prevent, detect or manage accidents and emergencies.

Many patients require monitoring for short or long periods of time. The reasons are manifold; some examples are: information gathering for the purpose of patient assessment (e.g. recording and analysis of the cardiac rhythm by means of the electrocardiogram in order to confirm diagnosis of arrhythmias); the immediate detection of medical emergencies signaled by changes in vital signs such as blood pressure or blood oxygen saturation or other biosignals, allowing rapid response when alarm conditions are raised; and routine measurements needed for management of chronic conditions (e.g. testing blood glucose in patients with diabetes mellitus). Nowadays the possibility of uploading sensor data over a telephone or Internet link means that patients who formerly had to be hospitalized for periods of monitoring can now be monitored at home. This alternative frees up hospital beds and staff (and thus reduces costs) and at the same time gives patients more freedom and better quality of life during periods of monitoring. Wireless communications bring the prospect of further freedom since in principle patients can now move freely anywhere in the world during monitoring, thanks to the development of wearable sensors and assuming global wireless coverage to enable transmission of biosignals.

In different situations different kinds of monitoring are appropriate. Some clinical conditions may call for signals to be measured without interruption and a continuous readout generated. An example would be measurement of blood pressure in a patient undergoing surgery, where BP is continuously measured by means of a catheter inserted into an artery. In other cases signals need to be sampled with a certain periodicity. Sampling rates can vary depending on the variable measured and the purpose of the monitoring. A typical sampling rate for oxygen saturation would be 5 times per second; for EMG sampling rates would be at least 2000 samples per second. This is also referred to as continuous monitoring. In other cases measurement may need to be done only on a daily or weekly basis.

The sampled biosignals may require real time processing. The processing may be done locally, as with a simple biofeedback device which gives real time auditory 
feedback and can be used to train patients to manage stress. Another example is the implanted cardiac pacemaker, which continuously measures heart rhythm and generates an electrical pulse in order to stimulate the cardiac muscle in case of too slow a heart rhythm. Another example is an automatic internal cardioverter/defibrillator (ICD), which acts if the heart rhythm exceeds a certain rate by delivering an electrical shock in order to restore the normal heart rhythm. These are examples of local processing of biosignals. If however the application is running remotely, at the hospital or health call center for example, then there may be a need for synchronous communications (generating an always on requirement for the communications). If post-hoc processing is sufficient then asynchronous communications will suffice and wireless communications will only be needed if there are other factors present (e.g. too much data is captured to be stored locally on the patient-worn equipment, or an alarm function is needed). An example of this is the continuous recording of heart rhythm over a period of several weeks for the diagnosis of infrequently occurring arrhythmias in patients with recurrent collapses using a recording device with limited data storage capacity. When a collapse occurs, data transmission through telephone lines from the device to the hospital enables evaluation of the cardiac rhythm during the collapse. Typically the always on feature is needed where a patient is at risk from sudden serious crises which would require rapid intervention. An example is the use of telemetry applied to in-patients with potentially lethal arrhythmias in the coronary care unit or on the ward. In the latter case, patients are free to walk around but their ECG is monitored continuously and instantaneously transmitted to a central control monitor. The monitor is observed around the clock by nurses trained to interpret the data and to act immediately in the event of a life threatening arrhythmia.

\section{BODY AREA NETWORKS FOR REMOTE MONITORING OF PATIENTS}

A wide range of patient monitoring equipment is used in healthcare. Some patient monitoring applications are described in another chapter of this book entitled MobiHealth: mobile health services based on body area networks.

Wearable monitoring devices are also marketed for use in various work or recreational settings to monitor a range of parameters. Use of heart rate monitors during fitness training is commonplace. Some devices incorporate motion sensors or environmental sensors. An example is the use of drop sensors and gas sensors for workers in hazardous environments such as chemical plants. An example of environmental sensors in recreation is the dive computer used by many scuba divers which displays parameters such as water temperature, current depth, maximum depth, dive time, pressure, ascent rate and time-to-fly. 
In the remainder of this section we introduce the concept of the Body Area Networks (BANs), and discuss the MobiHealth (MobiHealth, 2002) approach to remote monitoring.

\subsection{Body Area Networks}

We define a BAN as "a collection of (inter) communicating devices which are worn on the body, providing an integrated set of personalised services to the user" (WWRF, 2001). According to our definition a Body Area Network (BAN) incorporates a set of devices which perform some specific functions and which also perform communication. Communication amongst the elements of a BAN is called intra-BAN communication. If the BAN communicates externally, that is with other networks (which may themselves be BANs), this communication is referred to as extra-BAN communication.

\subsubsection{Intra-BAN Communications}

Intra-BAN communication may be transported over a wired or a wireless medium. Wired options include copper wires, optical fibres and various 'wearable computing' solutions. Wireless options include infrared light, microwave, radio and even skin conductivity.

\subsubsection{Extra-BAN Communications}

Extra-BAN communication may be based on wireless technologies. A range of wireless communication technologies is available including Bluetooth, WLAN, GSM, GPRS and UMTS. Bluetooth and WLAN (Wireless Local Area Network) are short and medium range communication technologies whilst GSM, GPRS and UMTS are wide area technologies, offering the possibility for the wearer of the BAN to roam freely outdoors within the coverage area.

\subsubsection{Body Area Networks for Healthcare}

The Body Area Network is a generic concept which can have many applications in various domains. When the devices of a BAN measure physiological signals or perform other actions for health-related purposes we can call this kind of specialization of Body Area Networks a health BAN. As a further specialisation, a BAN whose devices are biosignal sensors enables patient monitoring. If we combine health BANs with extra-BAN communications this enables remote monitoring of patients. In the following section we briefly describe the approach of the MobiHealth Project to the use of health BANs for remote monitoring of patients. 


\subsection{The MobiHealth Body Area Network}

The vision of the MobiHealth project was to develop an open extensible BAN platform which allows integration of different health functions by means of a plugand-play approach and thus to enable transmission of biosignals to a distant healthcare location. This enables remote management of chronic conditions and detection of health emergencies whilst giving the wearer the freedom to move anywhere in the world. Further details on the MobiHealth project and the MobiHealth BAN can be found in another chapter of this book entitled MobiHealth: mobile health services based on body area networks. An extension to the MobiHealth trauma application to scale up to disaster scenarios is described in another chapter in this section of the book entitled Telematics Support for Disaster Situations.

\subsubsection{Architecture}

The MobiHealth BAN consists of a central unit known as the MBU (Mobile Base Unit) and a set of devices, which may include sensors, actuators, and various multimedia devices. The MBU handles coordination, local computation and communication functions. The sensor data is processed by a sensor front-end before being transmitted to the MBU. A range of front-ends can be associated with an MBU, enabling customization of the BAN. The MBU currently used in the MobiHealth trials is based on the HP iPAQ platform. Future plans include porting to a cell phone platform such as the Sony Ericsson P800.

\subsubsection{Devices}

The generic BAN can be customized with different sensor sets for different applications. The main devices used in the MobiHealth trials were sensors, e.g. electrodes for measuring ECG, pulse oximeters for measuring oxygen saturation and calculating heart rate, sensors for measuring NIBP (non invasive blood pressure) and motion sensors.

\subsubsection{Intra-BAN Communications}

The MobiHealth approach allows for wired or wireless communications within the BAN, or a combination of the two. The BAN currently uses a combination of wired communication from sensors to sensor front end (later this could be replaced by smart sensors and wireless Zigbee technology) and Bluetooth.

\subsubsection{Extra-BAN Communications}


In the MobiHealth trials extra-BAN communication was via UMTS and GPRS. In many remote locations however the only alternative is satellite communications, since terrestrial links are not available everywhere.

\subsection{Clinical Settings in the MobiHealth Trials}

The overall goal of the MobiHealth project was to test the ability of 2.5 and $3 \mathrm{G}$ infrastructures to support value added services. Trials were conducted in four European countries in the areas of acute (trauma) care, chronic and high-risk patient monitoring and monitoring of patients in home-care settings. In this chapter we examine some possible applications of the MobiHealth concept for non-patients.

\subsection{Applicability in a Wider Context}

As well as serving patients, remote monitoring can also benefit non-patients going about their daily life activities. A special case of this is the use of monitoring to support work or sport in remote or extreme environments. In the following section we examine the role that monitoring can play in improving safety in extreme environments.

\section{MONITORING FOR HEALTH AND SAFETY IN EXTREME ENVIRONMENTS}

Extreme environments place unusual demands on equipment and human physiology, making even normal activities more complicated and difficult than usual. If people get into difficulties the evacuation process may be lengthy and complicated. Since activity in these environments is physically demanding, it is relatively simple to take preventative measures such as resting, if early warning of a problem can be given through monitoring. Monitoring and transmission of biosignals can also enable more sophisticated telemedical assistance. In this chapter we focus particularly on underwater and polar environments.

Extreme environments can be considered as environments in which one or more of the following characteristics apply: a) the environment imposes severe difficulties with respect to human existence, often requiring the aid of special equipment for survival and basic functioning; b) environmental conditions make equipment functioning and communication difficult; and c) evacuation of patients or casualties is made especially difficult because of environmental conditions. This definition includes the obviously extreme settings such as deep water diving, mountain climbing or crossing the ocean solo on a sailing boat. Our definition also covers extreme situations in daily communal life such as firefighters entering burning buildings (extreme by our definition due to the problems of evacuation, also extreme 
due to environmental conditions such as heat, poor vision and toxic gases), or even swimming in a swimming pool (extreme due to limits in the functioning of monitoring equipment and transmission of data underwater).

In the current chapter we focus on two of the more remote and severe environments (underwater and polar environments), since solutions fitting these cases can be adapted to less exotic situations.

\subsection{The Underwater Environment - Monitoring for Scuba Dvers}

Scuba divers face many health hazards including nitrogen narcosis, oxygen toxicity, decompression sickness and lung over-expansion injury. Monitoring of the diver's physiological status and transmission of biosignals to a remote medical facility can aid in prevention and management of diving accidents.

The underwater environment fits our definition of "extreme" on all three criteria: human existence is impossible without the supply of a breathing gas mixture (air, NitrOx, or other technical mixture), weights are required to remain submerged, a protective diving suit is almost always needed, and even movement and vision require special equipment (fins, mask). Operation of any electronic equipment is difficult, firstly due to the requirement for encasing it in a water-proof and pressureproof housing, and secondly due to the conductivity of the water which inhibits the use of some types of sensors. Underwater wireless communication is much more difficult than through the air and methods such as cabled communications or acoustic transmission are usually preferable. Evacuation of patients injured in diving operations is rarely easy. Since the breathing gas supply is limited the duration of the dive is planned and therefore known in advance. As a result notice of a problem underwater rarely takes more than an hour after its occurrence. Unfortunately, this type of "notification" (that is, the diver fails to surface within their known air capacity time) always means a serious and life threatening problem. Most divers do not carry any means of communication with the surface and rely on the assistance of their dive buddy.

Once a problem is detected, evacuation has three independent stages - getting the victim safely to the surface; carrying the victim to shore/boat and out of the water; and evacuating the victim to a medical center or a hospital capable of treating diving related problems. Even the last stage of the evacuation is complicated by the fact that, although air evacuation may be possible, the rescue aircraft must fly at very low altitudes to avoid (further) decompression effects on the casualty.

\subsubsection{The Underwater Environment and its Hazards for Human Physiology}

Human beings are poorly adapted for underwater activities and cannot survive without the aid of life support systems. Even with life support many of the diver's 
senses and capabilities are grossly impaired and a diver's ability to perform underwater is only a fraction of his/her ability on dry land.

The most dramatic effects of the underwater environment on human divers occur due to immersion and the rapid changes of ambient pressure which occur with depth. These two factors can cause a variety of related problems ranging in severity from mild to life threatening. Included among them are exposure and hypothermia, reduction of vital capacity of the respiratory system, over expansion of the lungs and other aspects of barotrauma (pressure injuries) and the various manifestations of decompression sickness (DCS).

At depth or under elevated ambient pressure, some of the gases breathed by divers dissolve into body tissue, in accordance with Henry's Law. During the decompression phase tissue becomes over-saturated with inert gas and the process of elimination of gas from the tissue takes place. Over-saturation beyond a certain value (critical over-saturation) can result in bubbles of inert gas forming in tissue and blood. The value of critical over-saturation as well as the rate of gas intake and elimination varies according to difference in tissue, and environmental and physiological factors.

During descent micro bubble formation does not occur, since all nitrogen is dissolved in the blood. It is during and after ascent that the micro bubbles emerge in the tissues and are transported through the blood to the lungs. These micro bubbles may cause local problem in joints, bones, and other tissue or through embolism in for instance the lungs or brain. Micro bubbles can be detected by ultrasound or doppler.

DCS has many different manifestations and is best categorized according to the organ or tissue effected. Brain, inner ear, spine, peripheral nerves, respiratory system, gastro-intestinal tract, musculoskeletal structure and skin are among the sites where DCS may manifest, with symptoms as variable as back pain, headache, apathy and malaise, and haematological changes. In most cases, the onset of symptoms will take place within six hours of ascent but there are well-recorded cases where symptoms of DCS have appeared after more than 24 hours. On the other hand, mostly in severe cases, symptoms may appear during ascent.

Over-expansion of the lungs (Pulmonary Barotrauma of ascent.) A diver using any kind of breathing apparatus is breathing gas at a pressure equal to the ambient pressure. By inhaling and exhaling continuously the pressure in the diver's respiratory system is constantly equalized to the ambient pressure, with no ill effects. This is true even if the ambient pressure is changing due to changes of depth. If however a diver fails to equalize the pressure in the lungs during ascent, because he holds his breath or due to various medical conditions which prevent adequate venting of excess gas through the bronchioles, the gas trapped in the lungs will expand. Expansion of gas in the respiratory system can result in stretching of alveoli at a first stage, which would be followed by alveoli rupture if pressure is not relieved. 
There are a number of phenomena associated with ruptured alveoli, all of which are dangerous, and some of which are severely life threatening. They are: inadequate gas exchange through the blood gas barrier in the alveoli due to damaged lung tissue, mediastinal and subcutaneous emphysema, pneumothorax and arterial gas embolism. These are among the most common diving related medical traumas.

The supply of oxygen to the diver has to be secured in order to survive and to perform labour, moreover the removal of carbon dioxide has to be secured. At greater depths, the use of compressed air is not suitable because of the risk of nitrogen narcosis, so other gases have to be used to avoid this problem.

Other risks to the diver are ear barotrauma, hypothermia, exhaustion, dehydration and decompression sickness. Other hazards result from currents, poor visibility, water pollution and toxic marine animals. Simple rupture of an eardrum can lead to vertigo and disorientation.

Depending on the situation, a diver may get his supply of oxygen from a surface station, and speech communication with the surface can be possible using full face masks and the umbilical which delivers the gas mixture. In many cases however, divers are self-supporting, carrying a limited amount of breathing gases, and communication may only be possible to another diver visually or, in case of poor visibility, through a line connecting two divers.

It is sometimes difficult to assess the actual condition of the diver. Especially in cases of nitrogen intoxication, the self assessment of the diver is impaired by a lowered and blurred consciousness. This can cause major judgment errors with possibly fatal outcome.

In addition to specific diving illnesses, the physical demands of diving and exposure to conditions which are malign to the diver - pressure, temperature, limited air supply - can trigger other medical events during a dive. For example heart attacks occur during diving with higher frequency than on land (comparing a population matched on health status, levels of activities, etc.). These events are considerably more deadly when occurring underwater than on land. This is simply because of the complications involved in detecting the problem and in fast but safe evacuation of the patient to a proper medical facility. Statistics on accidents and deaths show that cardiovascular events (heart attacks) cause 20 to 30 percent of all deaths which occur during scuba diving (source: Divers Alert Network). In most cases, early detection and termination of the dive could have prevented the event.

So, early warning to a diver of the possibility of an imminent medical situation can give the diver or his buddy the opportunity to take preventive measures. Early notification to the surface (to a diving monitoring post, a boat, or a shore base) of a diving emergency and of its nature, can trigger proper evacuation procedures which can mean the difference between life and death to the casualty.

The physiological measurements which would be most useful in order to assess the condition of the diver include heart rate, respiration rate, body temperature, oxygen and carbon dioxide content in the respiratory air, oxygen saturation and 
pressure and carbon dioxide pressure in the blood. Also useful would be blood pressure, dehydration level and microbubble formation.

\subsubsection{Sensors}

The obvious environmental conditions affecting underwater sensors are the wet (and sometimes saline) environment, temperature, pressure and poor visibility. These problems can be solved and many underwater sensors are in regular use by marine scientists engaged in environmental monitoring and as basic functions on existing dive computers. Divers are accustomed to carrying a considerable amount of gear and the diving suit itself provides a fairly stable framework or scaffold for positioning of sensors. The air-providing regulator, which is by definition in the mouth of the diver, provides another possible platform for sensors.

Due to the short duration of most dives, equipment does not require a long-life power supply and it is possible to recharge batteries between dives. Oxygen saturation level of haemoglobin can be measured optically. Positioning would aid location of the divers and could be transmitted in the form of a beaconing signal.

At the time of writing a few top-of-the-range dive computers offer breathing rate as one display feature. One wrist worn cardiac monitor targeted at the sports and training market is advertised as being water resistant to depth of 20 metres, but apparently has not been tested for divers.

\subsubsection{Communications}

Wireless communications are technically more difficult underwater than on land. Some short range wireless solutions are commercially available, for example wireless transmission of tank pressure from a tank mounted sensor to a wrist-worn dive computer. However the underwater environment limits the possibilities for long range wireless data communications at reasonable bandwidths. Wireless optical transmission is rather impractical underwater due to limitations on visibility. Low frequency radio is used by the military but requires high power and is not practical for recreational diving. Acoustic (audio) communication is the underwater wireless transmission mode of choice for recreational diving, offering limited bandwidth but good range. For voice communication various 'buddy phone' products are commercially available based on accoustic transmission (for example using ultrasonic, upper single sideband transmission). These can operate down to at least 40 metres in seawater with a range of anything up to 500 metres depending on sea conditions, and can be used diver to diver or diver to surface. However the price may be beyond the reach of many recreational divers. Data communications are a different story especially where transmission of high volume data such as certain biosiognal data or audio-video is required. If wired communication is possible this offers by far the best alternative for extra-BAN communication. A wired 
communication link can for instance be fully integrated with a diver's support line. This is not a problem where divers use surface supply breathing mixture (as is the case in many commercial diving operations), where an additional wire can be easily added to the diver's existing umbilical but it is not an option for divers using SCUBA (Self Contained Underwater Breathing Apparatus) gear. In cases where use of a cable is impossible or impractical, communication will need to be based on acoustic modulations and coding and compression of information. Such an acoustic signal could be received by a manned installation, such as a dive boat or a barge, or by a receiver attached to a surface buoy, where the signal could be converted to electromagnetic form and transmitted from the buoy through the air. Acoustic signals using low power high frequency transmitters have a relatively short range (in theory up to hundreds of metres, but usually tens of metres); hence there needs to be a receiving station within this range.

One of the limitations of acoustic systems is the difficulty of deriving accurate location information based on acoustic signals. Though such systems do exist, they are mainly deployed during accurate geographical surveys and other such operations requiring precise location control. Therefore the emergency function of the BAN system, the activation of which implies a call for immediate help, will need to produce a beaconing type acoustic signal. Obviously a matching detector will need to be available to the surface/rescue team. If a buoy based system can be used for relaying from the surface, a GPS location could be added to its transmissions topside. Diver carried portable location equipment is now commercially available which can operate when the diver is at the surface. It is intended to give divers short range communications so they can find their way back to a dive boat, or so the support team onboard can locate a diver in need of help. Surface voice communication systems to link divers in the water and surface support teams are also available. For longer range topside communication offshore the global solution is satellite transmission.

Location information is difficult underwater (GPS is not available underwater) but underwater location systems are available using a triangulation system, which requires (at least three) underwater installations at the dive location, in other words the systems relies on a fixed underwater infrastructure. This solution is suitable for certain kinds of dive sites where professional or recreational diving occurs regularly within the same area.

Intra-BAN communication is preferably integrated into the diving suit and other diver worn equipment. Wires (copper, optical fibre) are options. The special environmental factors of wetness, salinity and pressure have to be taken into account.

\subsection{Monitoring in Extreme Cold Environments}


Of the three criteria for extreme environments (severe hardship of human survival, on equipment functioning and communication, or on evacuation), polar regions present examples of two of the criteria and the Antarctic continent of all three; human survival requires special gear for constant protection as well as for performing the most simple of tasks. Communications are fairly straightforward in populated regions of the Arctic circle and often can rely on satellite coverage, but in more remote areas of the Arctic circle and in the Antarctic heavier systems using iridium satellites provide the backbone of communication. Evacuation can be very difficult, always requiring special equipment ranging from helicopters to specialised snow vehicles. Even so, weather conditions frequently prevent evacuation operations and during wintertime many field locations are totally cut off.

The extremely harsh climate of the world's polar regions imposes a spartan existence upon visitors, natives and local people, the land and sea based workers, mariners, scientists and others who live and operate there. The northern hemisphere polar regions, north of the artic circle, are sparsely populated with remote villages and towns, in contrast human activity in Antarctica is generally restricted to research stations and remote camps. However, with the increasing tourist movement in the Antarctic, and in agreement with the Antarctic Treaty, travel within the continent is rapidly expanding beyond the well-planned and monitored scientific expeditions to include also many groups of tourists and adventurers. Travel and research in these regions generally occurs during the summer months, which are characterized by 24 hour sunlight and warmer temperatures. During the dark winter months northern polar towns and villages remain active whilst in Antarctica the continental population plummets and 'winter-over' individuals are restricted to research stations.

The record for the coldest temperature on the earth's surface - $-89.6^{\circ} \mathrm{C}$ - was measured at Russia's Antarctic research station Vostok on July 21, 1983. The mean temperatures in the Antarctic interior range from $-40^{\circ} \mathrm{C}$ to $-70^{\circ} \mathrm{C}$ during the coldest month, and from $-15^{\circ} \mathrm{C}$ to $-32^{\circ} \mathrm{C}$ during the warmest month. On the coast, temperatures are considerably warmer: $-15^{\circ} \mathrm{C}$ to $-32^{\circ} \mathrm{C}$ in the winter, and from $+5^{\circ} \mathrm{C}$ to $-5^{\circ} \mathrm{C}$ in the summer, with the Antarctic Peninsula experiencing the highest temperatures year round.

Arctic regions are somewhat milder with average summer temperature in northern polar regions around $7.2^{\circ} \mathrm{C}$, and annual rainfall less than 4 inches; but nonetheless winter conditions and winter storms can halt any outdoor human activity.

The difficulty of evacuation from polar regions means that intervention via telemedicine can be vital, hence the potential value of communication of biosignals. Though primary importance should be given to monitoring vital signs and emergency related signals, a biomonitoring system which could measure and transmit a wide range of parameters is a vital prerequisite to remote surgery. The need for such remote surgical intervention was demonstrated in the tele-surgery performed in July 2002 at the South Pole research station, executed by the South Pole's physician with 
expert assistance given via a telemedicine link-up with Massachusetts General Hospital.

In cases where evacuation is needed, accurate positioning is needed for an evacuation team to locate the casualty. This could be via a beaconing/pinger type signal, however transmission of accurate GPS coordinates would be more useful for the long-range approach of the evacuation team.

\subsubsection{The polar environment and its hazards for human physiology}

The polar environment presents a range of challenges to human physiology. Hypothermia, simply defined as having a lower than normal temperature, is perhaps surprisingly not the most common hazard to human physiology in polar regions. It is, however, the easiest hazard which can be monitored via a sensor kit, primarily via measurement of core body temperature. A mildly hypothermic individual will have a temperature around $32.7-35^{\circ} \mathrm{C}$, while a profoundly hypothermic person will have a core body temperature of $32.2^{\circ} \mathrm{C}$ or lower. When the body's core temperature approaches $32.2^{0} \mathrm{C}$, the chance for core body systems failing increases. As core temperature drops, the body's muscular system becomes stiff and weak. The heart, being a muscle, reacts in the same way, and the number of beats per minute will begin to fall until, in a state of deep hypothermia, a heart rate of 20 beats per minute or less is not uncommon. Heart rate is an ancillary measurement that can indicate hypothermia.

To combat hypothermia, and to prepare for emergencies, people working in polar regions tend to carry a large amount of equipment, from protective gear to special support instrumentation. Carrying such a weight, in addition to the physical demands of moving and working in harsh conditions, can rapidly bring exhaustion and in extreme cases dehydration. In severe cases hypothermia can develop. Unfortunately, exhaustion and dehydration affect human physiology in synergy with hypothermia, hence exacerbating the patient's condition.

Since every venture outside shelter is physically demanding, any pre-existing medical conditions, and especially heart, lung, circulation and muscular problems, have a higher chance of manifesting. We have already seen that this is true also in the underwater environment.

The emergency related measurements we would like to monitor are divided into two categories: a) General distress signals, which should be measured, when possible, everywhere; b) Specific signals and vital signs that may warn of health related problem common to the specific environment at hand (in the polar region mainly hypothermia).

Of the first (general distress signals) one would like to measure blood pressure, heart rate, pulse shape, respiration/ventilation rate, dehydration level and temperature. Pulse shape would be useful as a trigger for immediate alarms, however it is not clear how this can be measured. Current devices work on heart rate. Nor is it 
clear how dehydration level can be measured. Hypothermia related signals are core body temperature and temperature of skin areas which are usually protected such as the chest and lower abdomen. In addition GPS positioning should be available for transmission at all times.

A BAN that is capable of transmitting emergency medical information may also be used for other medical situations. Stations in the polar regions are often isolated and therefore expert medical advice and telemedicine intervention can be critical. For such cases, small specialised sensor sets can be added to measure specific information. For example, in pregnancy and delivery situations, monitoring of contraction rate, maternal and foetal pulse rates, and of blood flow in amnionic cord or placenta would add important information if remote consultation is needed. In addition the system should be able to transmit audio and video signals from mobile and stationary medical facilities/ and also from vehicles.

\subsubsection{Sensors}

One function of the BAN is to alert its wearer of the approach or development of a potential medical emergency so that precautionary measures can be taken. If the situation reaches a critical stage then an alert to a medical alert and evacuation center needs to be transmitted. In such a case the exact location of the patient needs to be transmitted and in addition any medical information that will assist in the evacuation, and enable provision of first level medical treatment. The sensor sets and BAN for extreme cold environments can be the same as for the ordinary BAN applications, with the added proviso that battery packs should be of arctic specification.

\subsubsection{Communications}

The poles provide unique communication challenges. In both polar regions handheld radios, HF radios and satellite phones are available. The limitation of a handheld radio is the number of repeaters in position while HF radios require large antennas. Cellular phone technology is available is some parts of the northern polar region but coverage is inconsistent. Satellite communication could be used in both regions. The best solution would seem to be a combination of technologies based on satellite coverage but switching opportunistically to terrestrial technologies where available (this kind of solution is variously referred to as "beyond 3G", "3G and beyond" and "4G").

\section{CONCLUSIONS}


The use of Body Area Networks together with various wireless communication technologies offers one approach for remote monitoring. Convergence of communication and computation functionality, specifically seen in programmable cell phones and in pocket computers with telephony functionality, gives a choice of implementation platforms for Body Area Networks with extra BAN communications carried over (terrestrial) wireless telephony or via satellite links. As sensors get smaller and cheaper and self-powering devices become a reality, telemedicine services based on these technologies will become more viable and more convenient to use.

As well as serving patients in ordinary circumstances, remote monitoring has a special role to play in helping to maintain health, and even save life, for people who happen to live, work or play in various extreme environments.

\section{REFERENCES}

BlueTooth, 2003; http://www.bluetooth.org/

van Dam, K, S. Pitchers and M. Barnard, 'Body Area Networks: Towards a Wearable Future', Proc. WWRF kick off meeting, Munich, Germany, 6-7 March 2001; http://www.wireless-world-research.org/.

Jones, V. M., Bults, R. A. G., Konstantas, D., Vierhout, P. A. M., 2001a, Healthcare PANs: Personal Area Networks for trauma care and home care, Proceedings Fourth International Symposium on Wireless Personal Multimedia Communications (WPMC), Sept. 9-12, 2001, Aalborg, Denmark, http://wpmc01.org/, ISBN 87-988568-0-4

Jones, V. M., Bults, R. A. G., Konstantas, D., Vierhout, P. A. M,. 2001b, Body Area Networks for Healthcare, Proceedings Wireless World Research Forum meeting, Stockholm, 17-18 September 2001; http://www.wireless-world-research.org/

Konstantas, D., Jones, V. M., Bults, R. A. G., Herzog, R., 2001, MobiHealth innovative 2.5 / $3 \mathrm{G}$ mobile services and applications for healthcare, Thessaloniki.

Lymberis et al., Smart Mobile Applications for Health Professionals, 2003, IN MHealth: Emerging Mobile Health Systems, Robert H. Istepanian, Swamy Laxminarayan, Constantinos S. Pattichis, Editors, Kluwer Academic/Plenum Publishers. 
MobiHealth, 2002; http://www.mobihealth.org

Schmidt, R., 2001, Patients emit an aura of data, Fraunhofer-Gesellschaft, www.fraunhofer.de/english/press/md/md2001/md11-2001_t1.html

Widya, I., Vierhout, P. A. M., Jones, V. M., Bults, R. A. G., van Halteren, A., Peuscher, J. and Konstantas, D., Telematics Support for Disaster Situations, 2003, IN M-Health: Emerging Mobile Health Systems, Robert H. Istepanian, Swamy Laxminarayan, Constantinos S. Pattichis, Editors, Kluwer Academic/Plenum Publishers.

Wireless World Research Forum, 2001, The Book of Visions 2001: Visions of the Wireless World, Version 1.0, December 2001; http://www.wireless-worldresearch.org/

xMotion , 2002; http://www.ist-xmotion.org/

ZigBee Alliance, 2003; http://www.zigbee.org/

Zimmerman, T.G., 1999, 'Wireless networked devices: A new paradigm for computing and communication', IBM Systems Journal, Vol 38, No 4.

\section{ACKNOWLEDGEMENTS}

Our thanks go to the European Commission, who fund the MobiHealth project under the IST FP5 programme, and to the MobiHealth and xMotion partners. Thanks are also due for the support of: the University of Twente, (especially APS group, Department of Computer Science and CTIT); the Inter University Institute, Eilat, Israel; and Ben Gurion University, Beer Sheva, Israel, who supported Val Jones' sabbatical leave early in 2002 during which part of this research was conducted, thanks too to the many colleagues at UT, BGU and IUI who contributed knowledge, ideas and support during those three months. 